\title{
An Automatic Regularization Method : An Application for 3-D X-Ray Micro-CT Reconstruction Using Sparse Data
}

\section{Purisha, Zenith}

2019-02

Purisha, Z , Karhula , S S , Ketola , J H , Rimpeläinen , J , Nieminen , M T , Saarakkala , S , Kröger , H \& Siltanen , S 2019 , ' An Automatic Regularization Method : An Application for 3-D X-Ray Micro-CT Reconstruction Using Sparse Data ' , IEEE Transactions on Medical Imaging , vol. 38 , no. 2 , pp. 417-425 . https://doi.org/10.1109/TMI.2018.2865646

http://hdl.handle.net/10138/307032

https://doi.org/10.1109/TMI.2018.2865646

acceptedVersion

Downloaded from Helda, University of Helsinki institutional repository.

This is an electronic reprint of the original article.

This reprint may differ from the original in pagination and typographic detail.

Please cite the original version. 


\title{
An automatic regularization method: An application for 3D X-ray micro-CT reconstruction using sparse data
}

\author{
Zenith Purisha*, Sakari S. Karhula, Juuso H. Ketola, Juho Rimpeläinen, \\ Miika T. Nieminen, Simo Saarakkala, Heikki Kröger and Samuli Siltanen
}

\begin{abstract}
X-ray tomography is a reliable tool for determining the inner structure of $3 \mathrm{D}$ object with penetrating $\mathrm{X}$-rays. However, traditional reconstruction methods such as FDK require dense angular sampling in the data acquisition phase leading to long measurement times, especially in X-ray micro-tomography to obtain high resolution scans. Acquiring less data using greater angular steps is an obvious way for speeding up the process and avoiding the need to save huge data sets. However, computing 3D reconstruction from such a sparsely sampled dataset is difficult because the measurement data is usually contaminated by errors and linear measurement models do not contain sufficient information to solve the problem in practice. An automatic regularization method is proposed for robust reconstruction, based on enforcing sparsity in the three-dimensional shearlet transform domain. The inputs of the algorithm are the projection data and $a$ priori known expected degree of sparsity, denoted $0<\mathcal{C}_{p r} \leq 1$. The number $\mathcal{C}_{p r}$ can be calibrated from a few dense-angle reconstructions and fixed. Human subchondral bone samples were tested and morphometric parameters of the bone reconstructions were then analyzed using standard metrics. The proposed method is shown to outperform the baseline algorithm (FDK) in the case of sparsely collected data. The number of $X$-ray projections can be reduced up to $10 \%$ of the total amount 300 projections over 180 degrees with uniform angular step while retaining the quality of the reconstruction images and of the morphometric parameters.
\end{abstract}

\section{INTRODUCTION}

X-ray micro-tomography $(\mu \mathrm{CT})$ is an important tool in biomedical imaging and in industrial computed tomography. The principle of $\mathrm{X}$-ray $\mu \mathrm{CT}$ is to reveal the inner structure of an unknown object without destroying it by propagating $\mathrm{X}$-rays through the object. X-ray $\mu \mathrm{CT}$ will image an internal three-dimensional (3D) structure of the object at a high resolution.

In $\mu \mathrm{CT}$ a set of projection images is collected from many directions. A mathematical reconstruction algorithm is used

*Zenith Purisha, Juho Rimpeläinen and Samuli Siltanen are with the Department of Mathematics and Statistics, University of Helsinki, Finland. An asterisk indicates the corresponding author. E-mail: zenith.purisha@helsinki.fi

Zenith Purisha is with the Department of Mathematics, Universitas Gadjah Mada, Indonesia

Sakari S. Karhula is with Infotech Oulu, University of Oulu, Oulu, Finland

Sakari S. Karhula, Juuso H. Ketola, Miika T. Nieminen and Simo Saarakkala are with the Research Unit of Medical Imaging, Physics and Technology, University of Oulu, Oulu Finland

Miika T. Nieminen and Simo Saarakkala are with the Medical Research Center, University of Oulu and Oulu University Hospital, Oulu, Finland

Miika T. Nieminen and Simo Saarakkala are with the Department of Diagnostic Radiology, Oulu University Hospital, Oulu, Finland for revealing the 3D structure inside the sample. Conventional reconstruction methods such as Feldkamp-Davis-Kress type (FDK) [1] require densely sampled datasets to achieve sufficient reconstruction quality. More precisely, denote the projection angles by $\theta, 2 \theta, \ldots, N \theta=180$, with a fixed angular step $\theta>0$. The FDK algorithm typically needs at least 300 projections $(N=300)$ in order to deliver a high enough reconstruction quality. This often leads to the impractically of saving big data sets and long measurement times. In practice, quite often only a few X-ray $\mu \mathrm{CT}$ machines are available while the demand to get the acquisition data is high.

A simple way to speed up the acquisition process is to collect less data by decreasing $N$ and enlarging the angular step $\theta$ accordingly so that the 180 degree half-circle is still sampled. However, the task of computing a 3D reconstruction from such a sparsely sampled dataset becomes extremely sensitive to the data contaminated by errors and tomographic measurement model which does not have complete information. In mathematical terms, it is an ill-posed inverse problem [2] that needs to be regularized by making use of a priori information about the sample structure.

The shearlet transform is a tool for orientation-aware multiscale signal processing [3], [4]. Shearlets provide efficient representations for a variety of signals, see for example [5]. We implement a 3D tomographic reconstruction algorithm regularized by promoting the sparsity of the bone structure in the shearlet transform domain. We use an iterative soft thresholding algorithm, the so-called Primal Dual Fixed Point (PDFP) as outlined in [6]. The method was earlier introduced in [7]. Implementation of this method using wavelet transform as the penalty term has been successfully studied in [8].

We introduce a novel technique to make the regularized reconstruction process fully automatic. Namely, the PDFP method involves a thresholding parameter $\mu>0$. All shearlet coefficients smaller than $\frac{\mu}{2}$ in absolute value are set to zero in each iteration. How can a suitable value for $\mu$ be chosen?

If $\mu$ is large, then many coefficients vanish and the reconstruction is very sparse in the shearlet domain. If $\mu$ is small, then almost all shearlet coefficients of the final reconstruction will be nonzero. We propose determining the typical ratio $0<\mathcal{C}_{p r} \leq 1$ of nonzero shearlet coefficients from a few denseangle 3D reconstructions of both healthy and osteoarthritic bone samples. We let $\mu=\mu_{j}$ change in each iteration and apply a simple control algorithm so that $\mu_{j}$ converges to a limit 
value producing a reconstruction having the a priori known sparsity $\mathcal{C}_{p r}$. The integral controller, part of proportionalintegral-derivative controller (PID controller), is implemented in this approach [9].

Shearlet-based methods for X-ray tomography have been studied before, starting with [10] concentrating on inversion from noisy, densely sampled 2D sinograms. Total variation regularization and shearlet sparsity have been successfully combined for 2D tomographic data in [11], [12], including sparse data with a minimum of 128 angles. Shearlets have been shown to be useful for 2D region-of-interest tomography in [13] and for limited-angle tomography in [14]. For 3D X-ray tomography case, 3D-shearlet has been implemented in [15]. Regarding 3D tomography, the only shearlet study showing the optimality for representing tomographic data in terms of shearlets seems to be [16]. Research in shearlet-based regularization in sparse dynamic tomography has been studied in [15].

In this work, we investigate the reliability of a modern sparsity-promoting 3D reconstruction algorithm, controlled shearlet domain sparsity (CSDS), that promotes an a priori known level of sparsity and an adaptive method for choosing regularization parameter to reconstruct a standard phantom and human trabecular bone (healthy and OA) using sparse X-ray tomographic data. Particularly, we are accessing the thicknesses of the plates in the standard phantom [17]. For the bone sample, we quantify morphometric parameters of human trabecular bone calculated from $3 \mathrm{D}$ reconstructions $(e . g$ the percentage of bone volume $(B V / T V)$, trabecular thickness (Tb.Th) and trabecular separation (Tb.Sp)).These parameters are used for validation. They are important parameters to see the changes in the 3D structure of bone caused by osteoarthritis (OA), such as subchondral bone sclerosis [18], [19], [20], [21]. Also studies of osteoporotic bone have been done in [22], [23]. We use Computed Tomography Analyzer (CTAn) software to calculate the morphometric parameters from the trabecular bone. The parameters are defined for binary (bone/not-bone) $3 \mathrm{D}$ reconstructions; we use the Otsu algorithm in CTAn for segmentation [24]. A study to recover bone structure in 2D from sparse microtomography data using a tomographic method called the discrete algebraic reconstruction technique (DART) was introduced in [25], [26]. A bone study in liveanimal micro-CT imaging using DART has also been performed [27]. However, the method proposed here is based on different assumptions about a priori knowledge. DART needs rather accurate attenuation value estimates as input and assumes that the target consists of a small number of possible materials. The proposed algorithm does not need assumptions on how many materials are presents.

Reconstructions using 3D shearlet-sparsity regularization have been shown to outperform FDK in the case of sparsely collected data. With the shearlet-sparsity reconstruction method, the number of X-ray projections can be reduced to $10 \%$ of the 300 projections over 180 degrees while retaining the quality of morphometric analysis.

\section{Materials And Methods}

\section{A. 3D Tomographic Setup}

The goal of X-ray tomography is to recover the density function of an unknown object from measured projection data. In this paper, the object is three-dimensional, and cone-beam geometry is used for modelling the measurement.

Consider a physical domain $\Omega \subset \mathbb{R}^{3}$ and a non-negative $\mathrm{X}$-ray attenuation function $f: \Omega \rightarrow \mathbb{R}$. The X-rays travel through $\Omega$ along straight lines $L \subset \Omega$. After calibration, each pixel value in the collection of digital radiographs yields a line integral $\int_{L} f(x) d s$.

For computational reasons, a discrete model is required. Let us represent the attenuation values by a vector $\mathbf{f}=\left[\mathbf{f}_{i j k}\right] \in$ $\mathbb{R}^{N \times N \times T}$. Here $\mathbf{f}_{i j k}$ denotes the average of the values of the function $f$ over the voxel with indices $(i, j, k)$.

The line integral can be approximated by

$$
\int_{L} f(x) d s \approx \sum_{i=1}^{N} \sum_{j=1}^{N} \sum_{k=1}^{T} a_{i j k} \mathbf{f}_{i j k},
$$

where $a_{i j k}$ is a distance that the line $L$ travels in the voxel with indices $(i, j, k)$. Then the practical three-dimensional tomographic X-ray data is modelled by

$$
\mathbf{m}=\mathbf{A f}+\varepsilon
$$

with a matrix $\mathbf{A}$ containing one row for each pixel in the set of measurements and an additive noise $\varepsilon$.

We use the normalized measurement matrix $\frac{\mathbf{A}}{\|\mathbf{A}\|}$ and measurement data $\frac{\mathbf{m}}{\|\mathbf{A}\|}$. Note that the norm of $\mathbf{A}$ equals $\sqrt{\lambda}$, where $\lambda$ is the largest eigenvalue of the symmetric matrix $\mathbf{A}^{T} \mathbf{A}$. The power method can be used to compute $\lambda$ in a matrix-free fashion [28]. Multiplication by the matrices $\mathbf{A}$ and $\mathbf{A}^{T}$ can be implemented by using the SPOT operator [29].

\section{B. The Shearlet Transform}

Shearlets form a directional representation system for multidimensional data [30], [31]. They can overcome limitations of traditional systems like wavelets that only provide optimally sparse representations for functions of one variable. In the 3D case, shearlets offer optimal approximation of piecewise smooth functions with jumps appearing only along smooth surfaces.

Shearlets are parameterized by scale, shearing and translation indices organized in the set

$$
\Lambda=\mathbb{N}_{0} \times\left\{\left\lceil-2^{j / 2}\right\rceil, \ldots,\left\lceil 2^{j / 2}\right\rceil\right\} \times \mathbb{Z}^{3} .
$$

It has been shown in [3] that, under suitable assumptions, the collection $\psi_{\gamma}=\psi_{(l, m, n)} \in L^{2}\left(\mathbb{R}^{3}\right)$, where $\gamma=(l, m, n) \in \Lambda$, forms a frame for $L^{2}\left(\mathbb{R}^{3}\right)$ functions. The shearlet transform is defined as the following vector of coefficients:

$$
\mathcal{S}(\mathbf{f})=\left(\left\langle\mathbf{f}, \psi_{\gamma}\right\rangle\right)_{\gamma \in \Lambda} .
$$

We use the ShearLab implementation [4]. 


\section{Sparsity-promoting regularization}

In this work, we are interested in finding the vector $\mathbf{f}$ that minimizes the variational regularization functional

$$
\|\mathbf{A f}-\mathbf{m}\|_{2}^{2}+\mu \sum_{\gamma}\left|\left\langle\mathbf{f}, \psi_{\gamma}\right\rangle\right| .
$$

The parameter $\mu$ in (4) describes a trade-off between emphasizing either the data fidelity term or the regularizing penalty term.

We introduce a regularization method based on enforcing sparsity in the shearlet transform domain. In their seminal paper [6], Peijun Chen, Jianguo Huang, and Xiaoqun Zhang show that the minimizer of (4) can be computed using the primal-dual fixed point (PDFP) algorithm:

$$
\begin{aligned}
\mathbf{y}^{(i+1)} & =\mathbb{P}_{C}\left(\mathbf{f}^{(i)}-\tau \nabla g\left(\mathbf{f}^{(i)}\right)-\lambda \mathbf{S}^{T} \mathbf{v}^{(i)}\right) \\
\mathbf{v}^{(i+1)} & =\left(I-\mathcal{T}_{\mu}\right)\left(\mathbf{S} \mathbf{y}^{(i+1)}+\mathbf{v}^{(i)}\right) \\
\mathbf{f}^{(i+1)} & =\mathbb{P}_{C}\left(\mathbf{f}^{(i)}-\tau \nabla g\left(\mathbf{f}^{(i)}\right)-\lambda \mathbf{S}^{T} \mathbf{v}^{(i+1)}\right)
\end{aligned}
$$

where $\tau$ and $\lambda$ are positive parameters, $g(\mathbf{f})=\frac{1}{2}\|\mathbf{A f}-\mathbf{m}\|_{2}^{2}$, the matrix $\mathbf{S}$ is a digital implementation of the shearlet transform and $\mathcal{T}$ is the soft-thresholding operator defined by

$$
\mathcal{T}_{\mu}(c)= \begin{cases}c+\frac{\mu}{2} & \text { if } x \leq-\frac{\mu}{2} \\ 0 & \text { if }|x|<\frac{\mu}{2} \\ c-\frac{\mu}{2} & \text { if } x \geq-\frac{\mu}{2} .\end{cases}
$$

Here $\mu>0$ represents the thresholding parameter, while $\tau$ and $\lambda$ are parameters that need to be suitably chosen to guarantee convergence. In detail, $0<\lambda<1 / \lambda_{\max }\left(\mathbf{S S}^{T}\right)$, where $\lambda_{\max }$ denotes the maximum eigenvalue, and $0<\tau<2 / \tau_{\text {lip }}$, being $\tau_{\text {lip }}$ the Lipschitz constant for $g(\mathbf{f})$. Furthermore, in (5) the non-negative "quadrant" is denoted by $C=\mathbb{R}_{+}^{N^{2}}$ and $\mathbb{P}_{C}$ is the Euclidian projection. In other words, $\mathbb{P}_{C}$ replaces any negative elements in the input vector by zero.

\section{Automatic Selection of the Threshold Parameter $\mu$}

Assuming that we know a priori the expected degree of sparsity in the reconstruction, denoted as $\mathcal{C}_{p r}$, it is a positive value with the maximum value 1 . We use a simple feedback control system for finding such a value of $\mu$ that the iteration (5) produces a result with $\mathcal{C}_{p r} \cdot 100 \%$ of its shearlet coefficients nonzero.

In our proposed method, $\mu=\mu^{(i)}$ is allowed to vary during the iteration. Furthermore, it is automatically tuned in every iteration to:

$$
\mu^{(i+1)}:=\mu^{(i)}+\beta e^{(i+1)},
$$

where $e^{(i+1)}=\mathcal{C}^{(i)}-\mathcal{C}_{p r}$ and $0 \leq \mathcal{C}^{(i)} \leq 1$ is the sparsity level of the current iterate $\mathbf{f}^{(i)}$. If $\beta>0$ is too large, the controller induces oscillations in the regularization parameter and if it is too small convergence is slow. To avoid this, we choose a large initial value for beta, but decrease it each time the sparsity level crosesses the desired level of sparsity:

$$
\beta=\beta\left(1-\left|e^{(i+1)}-e^{(i)}\right|\right) .
$$

This approach can avoid unwanted oscillation in the values of $\mu^{(i)}$.

\section{E. Determining the A Priori Degree of Sparsity}

Denote $\mathbf{f}^{\kappa}$ as the best $\kappa$-term shearlet approximation using the $\kappa$ largest coefficients in the shearlets expansion [32]. We compute the best $\kappa$-term approximations of the baseline (FDK reconstruction from full projection images) image using different values of $\kappa$. Once we computed the images $\left\{\mathbf{f}^{\kappa}\right\}$ :

1) the morphometric parameters of trabecular bone (BV/TV, $T b . T h$ and $T b . S p$ ) for each images are computed using 3D analysis morphometry in CTAn software (see details in II-G) and

2) the plate thickness value for the standard phantom for each images are computed using 3D analysis morphometry as well.

At particular level, as the sparsity level $\kappa$ decreases, the morphometric parameters and the plate thickness value start to deteriorate. The prior sparsity level, $C_{p r}$ is chosen at the stage before at least one of the parameters in 1) and 2) for $\left\{\mathbf{f}^{\kappa}\right\}$ start to deteriorate.

\section{F. Pseudo-algorithm}

A step-by-step description of the proposed CSDS algorithm is summarized in Algorithm 1. As an addition, given $\mu \geq 0$, for a vector $s \in \mathbb{R}^{N^{2} \times T \times K}$, where $K$ is the number of $3 \mathrm{D}$ shearlets, we define the number of elements larger than $\mu$ in absolute value as follows:

$$
\#_{\mu} s:=\#\left\{i\left|1 \leq i \leq N^{2} \times T \times K,\right| s_{i} \mid>\mu\right\} .
$$

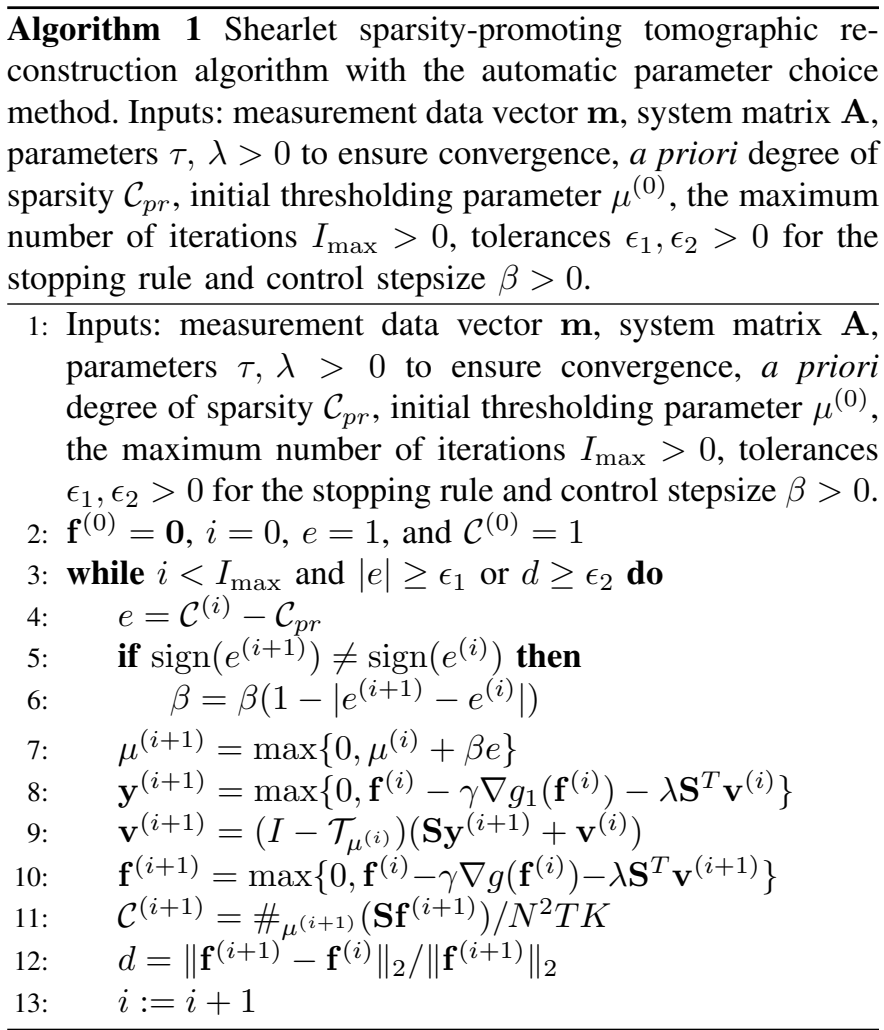




\section{G. Trabecular bone morphometric parameters}

We study human trabecular bone samples. The recommended parameters for studying the 3D structure of bone [18], [33], [34], [35] include:

1) Percentage of bone volume $(B V / T V)$. BV refers to volume of the region segmented as bone and $B V / T V$ refers to the ratio of the segmented bone volume to the total volume of the volume of interest (VOI);

2) Trabecular thickness (Tb.Th): the volume-based average of the maximum sphere thicknesses fitting in the structure $(\mu \mathrm{m})$

3) Trabecular separation ( $T b . S p)$ : the thickness of the spaces as defined by binarization within the VOI $(\mu \mathrm{m})$.

To calculate the basic bone morphometric parameters, standard Computed Tomography Analyzer (CTAn) software provided by the manufacturer (Bruker microCT, Kontich, Belgium) was used. The reconstructed images were converted to 8-bit images. Because the samples were drilled from bone, physical artifacts such as bone dust or cracks were left from the preparation. Therefore, a volume of interest (VOI) inside the sample was selected so that the edge artifacts would not affect the analysis. The VOI for the trabecular bone was $2.65 \times 2.65 \times 2.5 \mathrm{~mm}^{3}$ of the trabecular part in a square shape. Once the VOI of the images selected, images were then segmented into binary images using Otsu algorithm for morphometric analysis in CTAn. Pixels representing bone tissues were segmented as solid with gray-level values between 104 and 255. Despeckles algorithm (available in the software CTAn) is applied as well to remove speckles from the images.

We also tested the same steps to the phantom plates (see details in II-H1). In this case, each plate image was chosen separately as its VOI and Otsu algorithm used on each VOI to apply the segmentation. Pixels representing plates were segmented as solid with gray-level values between 184 and 255. Once the thresholding process is applied, the plate thickness is calculated in each VOI with CTAn as well [17].

\section{H. Experimental setup}

1) Phantom plates: X-ray measured data from a phantom which contains four different thickness of plates made of Aluminium placed in vertical positions. The thickness of the plates are 20, 50, 125 and $250 \mu \mathrm{m}$. The X-ray tomography was acquired with a SkyScan1272 high-resolution $\mu \mathrm{CT}$ scanner (Bruker microCT, Kontich, Belgium). The defaults of the rotation step are $0.8^{\circ}, 0.4^{\circ}$ or $0.2^{\circ}$ (which correspond to 225,450 and 900 projections over 180 degrees rotation, respectively. The scanning space: $75 \mathrm{~mm}$ in diameter and $70 \mathrm{~mm}$ in length.

The isotropic voxel size length for projection 22 is $\mu \mathrm{m} / \mathrm{pixel}$ and the number of frames averaged is 2 per projection. The projection images are collected as TIFF files and their dimensions are $1008 \times 672$. In the computation, a full binning was applied to the data. We collected 300 projection images acquired over a full 180 degree rotation with uniform angular step of 0.6 degrees between projections. Each projection image was composed of $1500 \mathrm{~ms}$ exposures. The X-ray tube acceleration voltage was $50 \mathrm{kV}$ and the tube current $200 \mu \mathrm{A}$. The full polychromatic beam was used for image acquisition. The

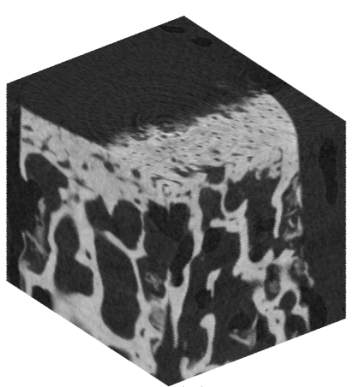

(a)

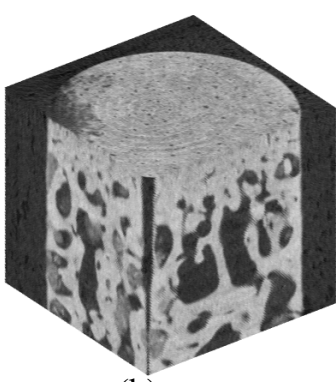

(b)
Fig. 1. 3D reconstructions of healthy (a) and osteoarthritis (b) human trabecular bone using FDK method from 300 projections.

additional filtration was $0.5 \mathrm{~mm}$ of Aluminium. We used 300 complete projections for baseline reconstructions. For sparse $\mathrm{x}$-ray reconstruction, we picked 30 and 50 projections from the measurement data (10\% and $17 \%$ from the 300 projections) with uniform angular sampling from different total opening angle of each projection image.

2) Human trabecular bone: X-ray data from two osteochondral samples were acquired. One healthy (bone-pathology free) bone sample (diameter $=4 \mathrm{~mm}$, height $=5 \mathrm{~mm}$ ) and one osteoarthritis $(\mathrm{OA})$ bone sample $($ diameter $=4 \mathrm{~mm}$, height $=$ $4 \mathrm{~mm}$ ) were harvested from the weight bearing area of tibial plateus from two cadavers under the approval of The Research Ethics Committee of the Northern Savo Hospital District, Kuopio, Finland (approval no 134/2015). Details of the data acquisition are set same as in II-H1, except the TIFF files (without binning process) are the input to compute the FDK and CSDS algorithms and their dimensions are $1008 \times 672$. We used 300 complete projections for baseline reconstructions. We picked two subsets of projections (30 and 50) from the measured data with uniform angular sampling from different total opening angles of each projection image.

\section{Morphometrics Parameters Values using Different Sparsity Levels}

The baseline $\mathbf{f}_{p r}$ was computed using the FDK method with complete projections.

A collection of $\kappa$-term approximation images, $\mathbf{f}_{p r}^{\kappa}$ were computed using the strategy discussed in Subsection II-E. The thresholded parameters $\kappa$ were selected from $95 \%$ to $5 \%$. This was done for the phantom plates as it is shown in Figure 2(a) and the bone samples (healthy and OA b) in Figure 1. The trabecular morphometrics parameters and plate thickness parameter of the phantom were calculated for each $\kappa$.

The plate thickness of the plates phantom starts to deteriorate for $\kappa \leq 45 \%$, while for $\kappa \leq 35 \%, B V / T V$ parameter of the trabecular bone starts to deteriorate (see Fig. S1 and S2 in Supplementary). Based on these results, we choose the $a$ priori degree of the sparsity level to be more than $35 \%$ for the human bone samples and more than $45 \%$ for the plates phantom. We denote the a priori degree of the sparsity level as $\mathcal{C}_{p r}$. 


\section{J. Image reconstruction}

The same size of reconstruction images were set for FDK and CSDS algorithms using a different number of projection images. The reconstruction images for plates phantom $120 \times 120 \times 100$ pixels $(=2.64 \times 2.64 \times 2.2 \mathrm{~mm})$ and for both trabecular samples have a size of $240 \times 240 \times 180$ pixels $(5.28 \times 5.28 \times 3.96 \mathrm{~mm})$.

For CSDS algorithm, the details are as follows: we used $\mathcal{C}_{p r}=50 \%$ for plates phantom and $\mathcal{C}_{p r}=37.5 \%$ for trabecular samples as it is explained in Subsection II-I. The initial value for the thresholding parameter $\mu^{0}$ was calculated from the absolute mean of $\left(1-\mathcal{C}_{p r}\right)$ of the shearlet coefficients from the backprojection reconstruction. The maximum number of iteration $I_{0}=1000$ (which is never attained in the results reported in section III) is set for additional termination criteria. However, in the computation, the number of iteration never reached the $I_{0}$.

We set the control step size $\beta=10 \mu^{0}, \epsilon_{1}=5 \times 10^{-3}$ and $\epsilon_{2}=1 \times 10^{-3}$ as the stopping rule. All the algorithms were implemented in Matlab 8.5. For FDK reconstructions, the experiments were performed on $\operatorname{Intel}(\mathrm{R}) \mathrm{Xeon}(\mathrm{R}) \mathrm{CPU}$ E5-1650 v3 at $3.7 \mathrm{GHz}$ RAM 32 and GPU 4GB memory. The CSDS method computation was performed on CPU at supercluster taito.csc.fi. The ASTRA Toolbox (iMinds-Vision Lab, University of Antwerp, Belgium) and Spot operator were implemented in reconstructions [29], [36], [37]. All of the computations were set up using a cone beam geometry.

The reconstructed images were converted to 8-bit images and then segmented into binary images for morphometric analysis in CTAn, Version 1.13.5.1 64-bit, SkyScan-Bruker microCT.

\section{RESULTS}

In this section, reconstruction images and calculation of trabecular morphometric parameters are presented.

\section{A. Plates phantom}

In Figure 2(a) shows the FDK reconstruction from complete (300) projections. The shearlet-based reconstruction is shown in Figure 2(c). The VOI of the reconstruction images were chosen for each plates from the top to the bottom of the plates reconstruction and segmented by applying the steps in Subsection II-G. For comparison, FDK reconstruction of plate phantom using 30 projections was computed as well as shown in Figure 2(b). Table I shows the thicknesses of each plates in the plate phantom. We only report the three thickest plates because the resolution is $22 \mu \mathrm{m}$ and the thinnest plate is only $20 \mu \mathrm{m}$ and the threshold value used for the binarization of the reconstructed images did not allow segmentation of the thinnest plate due to their high grey value [17]. The sparsity level for each iteration is presented in Figure 3.

\section{B. Human trabecular bone}

The shearlet-based reconstructions are shown in Figure 5. The sparsity level for each iteration is presented in Figure 6.
TABLE I

THE THICKNESS PARAMETERS $(T b . T h)$ CALCULATION FOR THE PLATES PHANTOM RECONSTRUCTIONS FROM 30 PROJECTION IMAGES.

\begin{tabular}{|r|c|c|c|c|}
\hline Method & $\begin{array}{c}\text { Number of } \\
\text { projections }\end{array}$ & $\begin{array}{c}\text { Plate 1 } \\
(\mu \mathrm{m})\end{array}$ & $\begin{array}{c}\text { Plate 2 } \\
(\mu \mathrm{m})\end{array}$ & $\begin{array}{c}\text { Plate 3 } \\
(\mu \mathrm{m})\end{array}$ \\
\hline \hline Baseline & 300 & 250 & 125 & 50 \\
\hline FDK & 30 & 443 & 267 & 142 \\
\hline CSDS & 30 & 320 & 171 & 88 \\
\hline
\end{tabular}

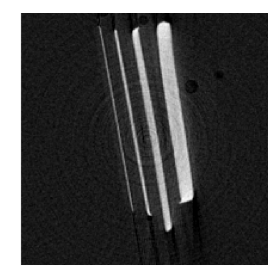

(a)

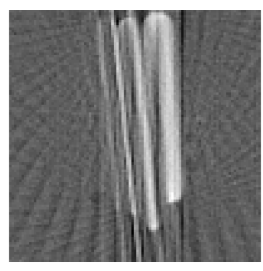

(b)

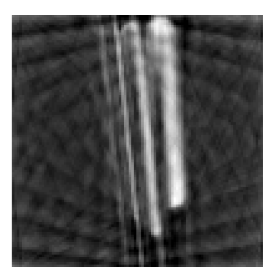

(c)
Fig. 2. Axial micro-CT cross-section images of the plates phantom reconstructions. The baseline image (FDK reconstruction from 300 projections) is given in (a), FDK reconstruction from 30 projections is shown in (b), CSDS reconstruction from 30 projections is shown in (c).

For comparison, FDK reconstruction of healthy and OA trabecular bone were computed as well as can be seen in Figure 5.

The VOI of the reconstruction images were chosen and segmented by applying the steps in Subsection II-G. The segmented images are shown in Figure 7. In addition, the trabecular bone morphometrics parameters for FDK and CSDS reconstructions were calculated and given in Table II.

\section{Discussion AND CONCLUSION}

The use of limited data is beneficial in reducing the often long scan times and avoiding massive amounts of data. It has also another advantage that is to avoid moving artifacts. While traditional methods such as FDK require dense projection images to produce good reconstructions, we propose the controlled shearlet domain sparsity method with automatically chosen regularization parameter for robust reconstruction for the incompletely sampled datasets. When the number of projection images was reduced, the significant streak artifacts overwhelm the FDK reconstruction images while the CSDS reconstructions contain less streak artifacts. The non-negativity constraint and the enforcement of the penalty term $\ell_{1}$-norm combined with the sparsity transform which acts as a denoising

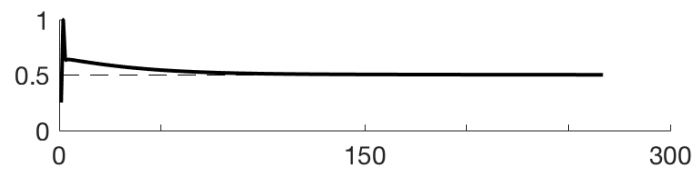

Fig. 3. The ratio of nonzero shearlet coefficients of reconstructing plates phantom using 30 projections as the iteration progresses (thick line). The dashed line is the $C_{p r}$. The iterations number is 267 and the computation time is 33637 seconds. 


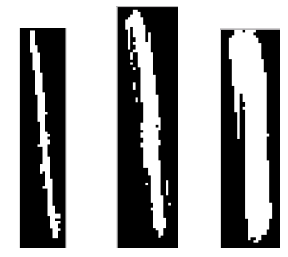

(a)

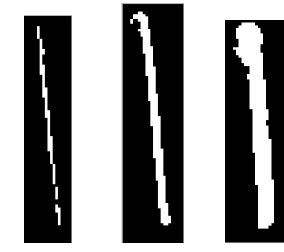

(b)
Fig. 4. (a) and (b) Axial micro-CT cross-section images of thresholded images or binary images of the three thickest plates phantom reconstructions correspond to Figure 2(b) and 2(c), respectively.

TABLE II

THE TRABECULAR BONE MORPHOMETRIC PARAMETERS CALCULATION FOR THE HEALTHY AND OA SAMPLES RECONSTRUCTION FROM DIFFERENT NUMBER OF PROJECTION IMAGES.

\begin{tabular}{l}
\begin{tabular}{|r|c|c|c|c|}
\hline Method & $\begin{array}{c}\text { Number of } \\
\text { projections }\end{array}$ & $B V / T V$ & $\begin{array}{c}\text { Tb.Th } \\
(\mu \mathrm{m})\end{array}$ & $\begin{array}{c}\text { Tb.Sp } \\
(\mu \mathrm{m})\end{array}$ \\
\hline \hline Baseline & 300 & $32.33 \%$ & 340 & 710 \\
\hline FDK & 50 & $30.50 \%$ & 280 & 640 \\
& 30 & $32.17 \%$ & 240 & 510 \\
\hline CSDS & 50 & $33.77 \%$ & 360 & 700 \\
& 30 & $34.29 \%$ & 330 & 630 \\
\hline Baseline & 300 & $51.30 \%$ & 370 & 350 \\
\hline FDK & 50 & $50.60 \%$ & 300 & 290 \\
& 30 & $48.57 \%$ & 210 & 210 \\
\hline CSDS & 50 & $53.69 \%$ & 360 & 310 \\
& 30 & $52.79 \%$ & 370 & 330 \\
\hline
\end{tabular} \\
\hline
\end{tabular}

process in the CSDS method give significant contribution to produce better reconstructions as is shown in Figure 2 and 5.

Figure 3 and 6 presents the behaviors of the sparsity levels for each datasets. In the initial iterations, short oscillations appear due to the large value of the tuning parameter $\beta$. They soon disappear as the 'crossing' checking process decreases the parameter, as discussed in Section II-D and eventually the ratio of nonzero coefficients, $\mathcal{C}_{i}$ converges to $\mathcal{C}_{p r}$. This is one of the benefits of the CSDS proposed method, as the manual tuning could be avoided.

TABLE III

COMPUTATION TIMES OF 3D RECONSTRUCTION USING FDK METHOD (IN SECONDS)

\begin{tabular}{|c|c|}
\hline Number of projections & FDK for healthy and OA samples \\
\hline \hline 50 & 5.0 \\
30 & 3.0 \\
\hline
\end{tabular}

TABLE IV

COMPUTATION TIMES OF 3D RECONSTRUCTION USING SHEARLET-BASED METHOD FOR HEALTHY AND OA SAMPLES (IN SECONDS)

\begin{tabular}{|c|c|c|}
\hline $\begin{array}{c}\text { Number of } \\
\text { projections images }\end{array}$ & $\begin{array}{l}\text { shearlet-based method } \\
\text { for healthy bone }\end{array}$ & $\begin{array}{l}\text { shearlet -based method } \\
\text { for OA bone }\end{array}$ \\
\hline $\begin{array}{l}50 \\
30\end{array}$ & $\begin{array}{c}64001 \\
\text { (256 iterations) } \\
46642 \\
\text { (183 iterations) }\end{array}$ & $\begin{array}{c}60768 \\
\text { (292 iterations) } \\
47715 \\
\text { (190 iterations) }\end{array}$ \\
\hline
\end{tabular}

Besides the visual inspection, in this particular problem, we also measured the quality of the reconstructions quantitatively. We computed the morphometric parameters of the reconstructions, and compare them to the parameters from the baseline images.

The CSDS reconstruction shows that the three thickest plates are sharper than the FDK reconstruction. The computations of morphometric parameters were done by using the standard steps in II-G. The plates thickness of the phantom from CSDS reconstruction yield less deviation against the target thickness: $28 \%-76 \%$ compared to the FDK reconstruction for which the deviation was $77.2 \%-184 \%$. It could be seen in Figure 4 that binarised images of the plates using CSDS algorithm are better than using FDK.

In the FDK reconstruction from undersampled data, the quality of the binary images were relatively poor since many speckles induced by the noise randomly appeared in the binary images. It can be seen in Figure 7 that many of trabeculae were also broken. As a result, trabecular bone morphometric parameters progressively showed considerable differences when fewer projection images was used compared to the baseline (full projection images). For instance, for both samples, the FDK reconstructions using 50 projections had differences in the $T b . S p$ parameter of $5.5 \%-17.1 \%$, while for 30 projections it was $25.5 \%-40 \%$. Other parameters such as Tb.Th was affected significantly as well for the two different numbers of projections. The $T b . T h$ decreased by up to $43.2 \%$ of its baseline value (from $370 \mu \mathrm{m}$ to $210 \mu \mathrm{m}$ or from 340 $\mu \mathrm{m}$ to $240 \mu \mathrm{m})$. In addition, since threshold values of the phantom plates and trabecular bone indicated in II-G are quite different, it seems to affect the measured thickness parameters in an opposite trend to both plates and the bone (the Tb.Th of the plates increases with sparser number of projections and it decreases in the bone sample as shown in Table I and II).

The preliminary results from the CSDS algorithm show that the differences in the parameters are relatively smaller than those of the FDK method. For instance Tb.Sp increased by up to $14.6 \%$ difference of its baseline value for 50 projections and $5.7 \%-10.9 \%$ for 30 projections. This is due to the absence of noise speckles in the binary images shown in Figure 7. It is reported as well that $\mathrm{Tb}$.Th for 50 projections increased only up to $5.88 \%$, and $2.94 \%$ decreased for 30 projections.

The $B V / T V$ increased by a relatively small amount: from CSDS method it increased up to $4.7 \%$, not significant difference compared to the FDK method for which the deviation was up to $5.3 \%$.

Finally, reduction of the number of projections had less significant effects on the binary images of the CSDS method. The appearance of the speckles noise was insignificant even for a small number of projection images. Therefore, better results in the thresholded images were obtained. As we can see in Table II, the trabecular bone morphometric parameters calculation $(B V / T V, T b . T h$, and $T b . S p)$ for healthy and OA samples using sparser projection images is relatively closer to the values of the baseline parameters. Some works related to subchondral bone 3D microarchitecture in human has been studied in [38], and for OA bone it has been done in [39]. In this work, the $B V / T V$ and $T b . S p$ values for OA sample are in 


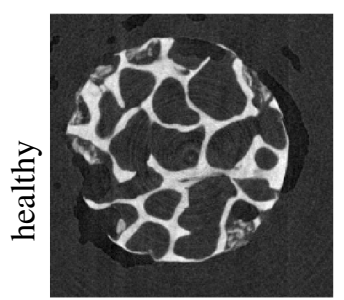

(a)

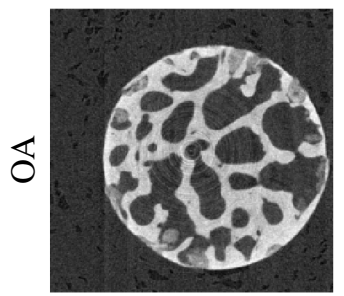

(f)

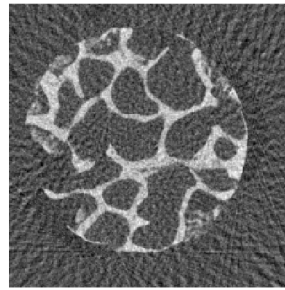

(b)

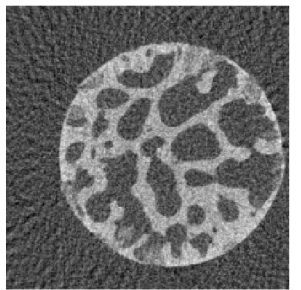

(g)

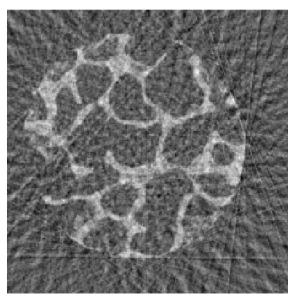

(c)

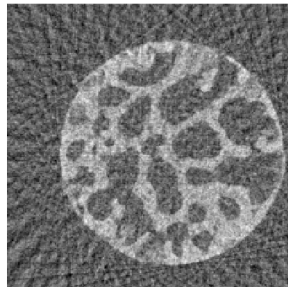

(h)

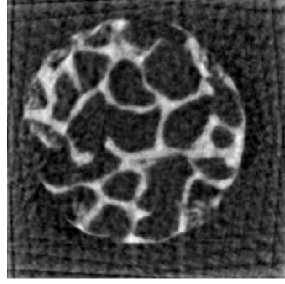

(d)

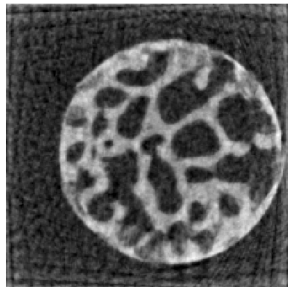

(i)

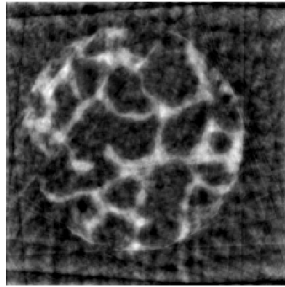

(e)

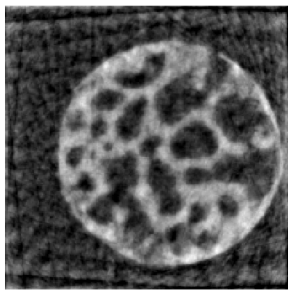

(j)

Fig. 5. Axial micro-CT cross-section images of the trabecular bone reconstructions. The baseline images (FDK reconstruction from 300 projections) are given in (a) and (f), FDK reconstructions from 50 projections are shown in (b) and (g), FDK reconstructions from 30 projections are shown in (c) and (h), CSDS reconstructions from 50 projections are shown in (d) and (i) and CSDS reconstructions from 30 projections are shown in (e) and (j). FDK reconstructions are overwhelmed by streak artifacts. The artifacts in CSDS reconstructions are less dominant but the edges seem to smooth out.

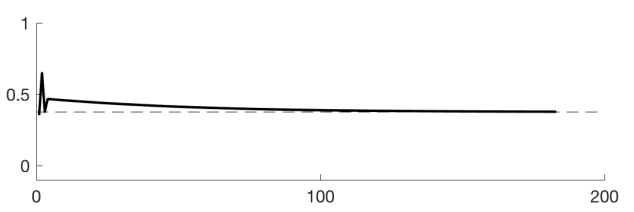

(a)

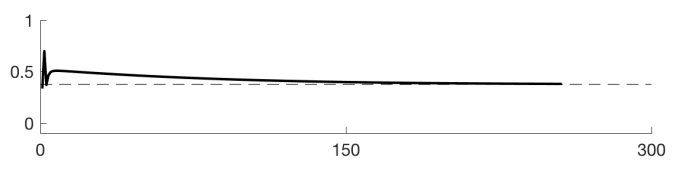

(b)

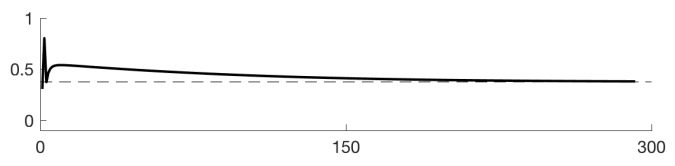

(c)

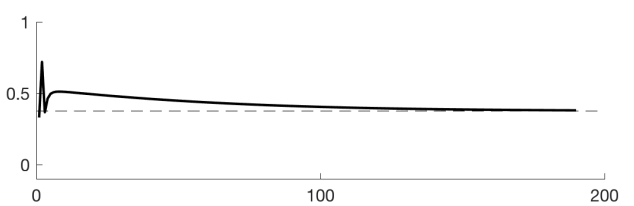

(d)

Fig. 6. The ratio of nonzero shearlet coefficients as the iteration progresses (thick line). The dashed line is the $C_{p r}$ (a) and (b): the healthy sample using 30 and 50 projections. (c) and (d): the OA sample using 30 and 50 projections the average of statistical data reported in [39], and the $T b . T h$ is at around the maximum values in the statistical data.

The results show that implementing the CSDS approach to reconstruct the inner structure of the samples using considerably sparse projection images outperforms the conventional FDK approach. The CSDS reconstructions seem to be smoothing out the edges as shown in Figure 5, however by increasing the scale parameter in the shearlet transform, we should be able to capture more details of the image.

Despite its success, the computational burden of the CSDS method is relatively high (up to 17.7 hours). It is also heavier than DART algorithm which only took less than 10 minutes. However, the computation time could be sped-up by implementing parallelized GPU code. Another acceleration strategy is to compute the shearlet decomposition in a serialized manner so that one does not need to keep all shearlet coefficients in memory at the same time. The computation times of the FDK and the shearlet based method using different number of projection images are shown in Tables IV and III.

In this study, there is no statistical comparisons because in fact collecting in vitro samples from patients is relatively hard and time consuming. Also the size of the samples is limited. Therefore, the results presented here were reported as a preliminary study. However, for the future work, statistical analysis in comparing the morphometric variables for each samples might be also considered. The range of a priori sparsity level $\mathcal{C}_{p r}$ from more data could also be computed. Applying the method in vivo would be also interesting to do as a future research as high ionizing radiation doses in $\mu \mathrm{CT}$ in in vivo experiment could be reduced. It has been discussed that high radiation could increase the risk of cancer, birth defects 


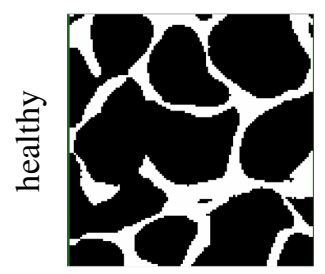

(a)

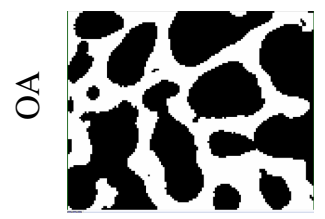

(f)

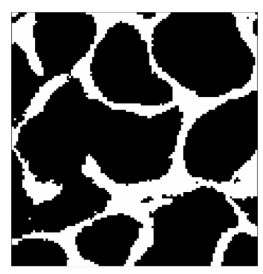

(b)

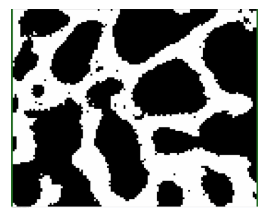

(g)

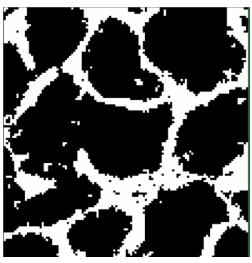

(c)

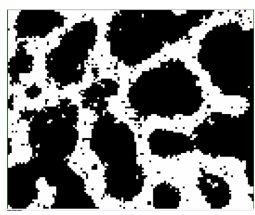

(h)

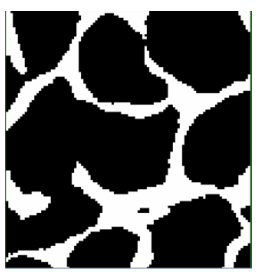

(d)

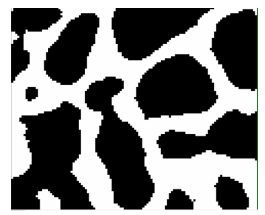

(i)

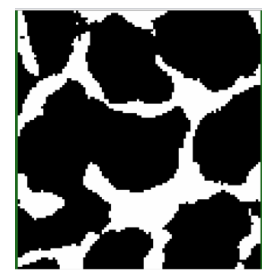

(e)

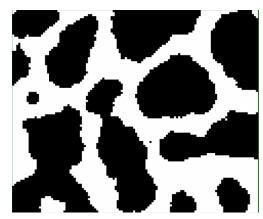

(j)

Fig. 7. Axial cross-section images of thresholded images or binary images of the volume of interest which correspond to Figure 5.

or heritable mutations [40], [41].

\section{ACKNOWLEDGEMENTS}

This work was supported by the Academy of Finland (Finnish Centre of Excellence in Inverse Problems Research 2012-2017, and grant nos. 268378 and 303786), and the European Research Council under the European Unions Seventh Framework Programme (FP/2007-2013)/ERC Grant Agreement no. 336267. We warmly thank Maximilian März and Tatiana Bubba for discussions about the shearlets. The authors also wish to acknowledge CSC-IT Center for Science, Finland, for computational resources.

\section{REFERENCES}

[1] L. Feldkamp, L. Davis, and J. Kress, "Practical cone-beam algorithm," JOSA A, vol. 1, no. 6, pp. 612-619, 1984.

[2] J. L. Mueller and S. Siltanen, Linear and nonlinear inverse problems with practical applications. Siam, 2012, vol. 10.

[3] G. Kutyniok and D. Labate, Shearlets: Multiscale analysis for multivariate data. Springer Science \& Business Media, 2012.

[4] G. Kutyniok, W.-Q. Lim, and R. Reisenhofer, "Shearlab 3d: Faithful digital shearlet transforms based on compactly supported shearlets," ACM Transactions on Mathematical Software (TOMS), vol. 42, no. 1, p. 5, 2016.

[5] K. Guo and D. Labate, "Optimally sparse representations of 3d data with $c^{2}$ surface singularities using parseval frames of shearlets," SIAM Journal on Mathematical Analysis, vol. 44, no. 2, pp. 851-886, 2012. [Online]. Available: http://dx.doi.org/10.1137/100813397

[6] P. Chen, J. Huang, and X. Zhang, "A primal-dual fixed point algorithm for minimization of the sum of three convex separable functions," Fixed Point Theory and Applications, vol. 2016, no. 1, p. 54, 2016.

[7] I. Daubechies, M. Defrise, and C. De Mol, "An iterative thresholding algorithm for linear inverse problems with a sparsity constraint," Communications on pure and applied mathematics, vol. 57, no. 11, pp. 1413-1457, 2004.

[8] Z. Purisha, J. Rimpeläinen, T. Bubba, and S. Siltanen, "Controlled wavelet domain sparsity for x-ray tomography," Measurement Science and Technology, vol. 29, no. 1, p. 014002, 2017.
[9] K. J. Åström and T. Hägglund, "Pid controllers: theory, design, and tuning," 1995.

[10] F. Colonna, G. Easley, K. Guo, and D. Labate, "Radon transform inversion using the shearlet representation," Applied and Computational Harmonic Analysis, vol. 29, no. 2, pp. 232-250, 2010.

[11] B. Vandeghinste, B. Goossens, R. Van Holen, C. Vanhove, A. Pizurica, S. Vandenberghe, and S. Staelens, "Combined shearlet and tv regularization in sparse-view ct reconstruction," in 2nd International Meeting on image formation in X-ray Computed Tomography, 2012.

[12] E. Garduño and G. T. Herman, "Computerized tomography with total variation and with shearlets," arXiv preprint arXiv:1608.06668, 2016.

[13] T. Bubba, F. Porta, G. Zanghirati, and S. Bonettini, "The roi ct problem: a shearlet-based regularization approach," in Journal of Physics: Conference Series, vol. 756, no. 1. IOP Publishing, 2016, p. 012009.

[14] J. Frikel, "Sparse regularization in limited angle tomography," Applied and Computational Harmonic Analysis, vol. 34, no. 1, pp. 117-141, 2013.

[15] T. A. Bubba, M. März, Z. Purisha, M. Lassas, and S. Siltanen, "Shearlet-based regularization in sparse dynamic tomography," Proc. SPIE, vol. 10394, pp. 10394 - 10394 - 10, 2017. [Online]. Available: http://dx.doi.org/10.1117/12.2273380

[16] K. Guo and D. Labate, "Optimal recovery of 3d x-ray tomographic data via shearlet decomposition," Advances in Computational Mathematics, vol. 39, no. 2, pp. 227-255, 2013.

[17] E. Perilli, F. Baruffaldi, M. Bisi, L. Cristofolini, and A. Cappello, "A physical phantom for the calibration of three-dimensional $\mathrm{x}$-ray microtomography examination," Journal of microscopy, vol. 222, no. 2, pp. 124-134, 2006.

[18] M. L. Bouxsein, S. K. Boyd, B. A. Christiansen, R. E. Guldberg, K. J. Jepsen, and R. Müller, "Guidelines for assessment of bone microstructure in rodents using micro-computed tomography," Journal of bone and mineral research, vol. 25, no. 7, pp. 1468-1486, 2010.

[19] A. Postnov, A. Vinogradov, D. Van Dyck, S. Saveliev, and N. De Clerck, "Quantitative analysis of bone mineral content by x-ray microtomography," Physiological measurement, vol. 24, no. 1, p. 165, 2003.

[20] M. A. Finnilä, J. Thevenot, O.-M. Aho, V. Tiitu, J. Rautiainen, S. Kauppinen, M. T. Nieminen, K. Pritzker, M. Valkealahti, P. Lehenkari et al., "Association between subchondral bone structure and osteoarthritis histopathological grade," Journal of Orthopaedic Research, 2016.

[21] G. Mohan, E. Perilli, J. S. Kuliwaba, J. M. Humphries, I. H. Parkinson, and N. L. Fazzalari, "Application of in vivo micro-computed tomography in the temporal characterisation of subchondral bone architecture in a rat model of low-dose monosodium iodoacetate-induced osteoarthritis," Arthritis research \& therapy, vol. 13, no. 6, p. R210, 2011. 
[22] R. D. Kapadia, G. B. Stroup, A. M. Badger, B. Koller, J. M. Levin, R. W. Coatney, R. A. Dodds, X. Liang, M. W. Lark, and M. Gowen, "Applications of micro-ct and $\mathrm{mr}$ microscopy to study pre-clinical models of osteoporosis and osteoarthritis," Technology and Health Care, vol. 6, no. 5, 6, pp. 361-372, 1998.

[23] R. Müller, H. Van Campenhout, B. Van Damme, G. Van der Perre, J. Dequeker, T. Hildebrand, and P. Rüegsegger, "Morphometric analysis of human bone biopsies: a quantitative structural comparison of histological sections and micro-computed tomography," Bone, vol. 23, no. 1, pp. 59-66, 1998.

[24] N. Otsu, "A threshold selection method from gray-level histograms," Automatica, vol. 11, no. 285-296, pp. 23-27, 1975.

[25] K. Batenburg and J. Sijbers, "Discrete tomography from micro-ct data: application to the mouse trabecular bone structure," in Medical Imaging. International Society for Optics and Photonics, 2006, pp. 614240 614240.

[26] K. J. Batenburg and J. Sijbers, "Dart: a practical reconstruction algorithm for discrete tomography," IEEE Transactions on Image Processing, vol. 20, no. 9, pp. 2542-2553, 2011.

[27] E. Van de Casteele, E. Perilli, W. Van Aarle, K. J. Reynolds, and J. Sijbers, "Discrete tomography in an in vivo small animal bone study," Journal of Bone and Mineral Metabolism, pp. 1-14, 2017.

[28] G. H. Golub and C. F. Van Loan, Matrix computations. JHU Press, 2012, vol. 3.

[29] F. Bleichrodt, T. van Leeuwen, W. J. Palenstijn, W. van Aarle, J. Sijbers, and K. J. Batenburg, "Easy implementation of advanced tomography algorithms using the astra toolbox with spot operators," Numerical Algorithms, vol. 71, no. 3, pp. 673-697, 2016.

[30] K. Guo, G. Kutyniok, and D. Labate, "Sparse multidimensional representations using anisotropic dilation and shear operators," Wavelets und Splines (Athens, GA, 2005), G. Chen und MJ Lai, eds., Nashboro Press, Nashville, TN, pp. 189-201, 2006.

[31] D. Labate, W.-Q. Lim, G. Kutyniok, and G. Weiss, "Sparse multidimensional representation using shearlets," in Optics \& Photonics 2005. International Society for Optics and Photonics, 2005, pp. 59140U$59140 \mathrm{U}$.

[32] R. A. DeVore, "Nonlinear approximation," Acta numerica, vol. 7, pp. 51-150, 1998.

[33] T. Hildebrand and P. Rüegsegger, "A new method for the modelindependent assessment of thickness in three-dimensional images," Journal of microscopy, vol. 185, no. 1, pp. 67-75, 1997.

[34] A. M. Parfitt, M. K. Drezner, F. H. Glorieux, J. A. Kanis, H. Malluche, P. J. Meunier, S. M. Ott, and R. R. Recker, "Bone histomorphometry: standardization of nomenclature, symbols, and units: report of the asbmr histomorphometry nomenclature committee," Journal of bone and mineral research, vol. 2, no. 6, pp. 595-610, 1987.

[35] F. Marinozzi, A. Marinozzi, F. Bini, F. Zuppante, R. Pecci, and R. Bedini, "Variability of morphometric parameters of human trabecular tissue from coxo-arthritis and osteoporotic samples," Annali dell'Istituto superiore di sanita, vol. 48, no. 1, pp. 19-25, 2012.

[36] W. van Aarle, W. J. Palenstijn, J. Cant, E. Janssens, F. Bleichrodt, A. Dabravolski, J. De Beenhouwer, K. J. Batenburg, and J. Sijbers, "Fast and flexible x-ray tomography using the astra toolbox," Optics Express, vol. 24, no. 22, pp. 25 129-25 147, 2016.

[37] W. van Aarle, W. J. Palenstijn, J. De Beenhouwer, T. Altantzis, S. Bals, K. J. Batenburg, and J. Sijbers, "The astra toolbox: A platform for advanced algorithm development in electron tomography," Ultramicroscopy, vol. 157, pp. 35-47, 2015.

[38] M. Ding and I. Hvid, "Quantification of age-related changes in the structure model type and trabecular thickness of human tibial cancellous bone," Bone, vol. 26, no. 3, pp. 291-295, 2000.

[39] B. C. Roberts, D. Thewlis, L. B. Solomon, G. Mercer, K. J. Reynolds, and E. Perilli, "Systematic mapping of the subchondral bone $3 \mathrm{~d}$ microarchitecture in the human tibial plateau: variations with joint alignment," Journal of Orthopaedic Research, vol. 35, no. 9, pp. 1927-1941, 2017.

[40] D. J. Brenner, R. Doll, D. T. Goodhead, E. J. Hall, C. E. Land, J. B. Little, J. H. Lubin, D. L. Preston, R. J. Preston, J. S. Puskin et al., "Cancer risks attributable to low doses of ionizing radiation: assessing what we really know," Proceedings of the National Academy of Sciences, vol. 100, no. 24, pp. 13 761-13766, 2003.

[41] R. Mitchel, "Low doses of radiation reduce risk in vivo," Dose-Response, vol. 5, no. 1, pp. dose-response, 2007. 